\title{
OPTICAL INSTRUMENTS SYNERGY IN DETERMINATION OF OPTICAL DEPTH OF THIN CLOUDS
}

\author{
Daniela Viviana Vlăduțescu ${ }^{1}$, Stephen E. Schwartz ${ }^{2}$, Dong Huang ${ }^{3}$ \\ ${ }^{I}$ New York City College of Technology of the City University of New York, USA \\ vivianavladutescu@yahoo.com, \\ ${ }^{2}$ Environmental and Climate Sciences, Brookhaven National Laboratory, NY, USA \\ ${ }^{3}$ Science Systems and Applications Inc., Greenbelt, MD, USA
}

\begin{abstract}
Optically thin clouds have a strong radiative effect and need to be represented accurately in climate models. Cloud optical depth of thin clouds was retrieved using high resolution digital photography, lidar, and a radiative transfer model. The Doppler Lidar was operated at $1.5 \mu \mathrm{m}$, minimizing return from Rayleigh scattering, emphasizing return from aerosols and clouds. This approach examined cloud structure on scales 3 to 5 orders of magnitude finer than satellite products, opening new avenues for examination of cloud structure and evolution.
\end{abstract}

\section{INTRODUCTION}

Considering the substantial influence of clouds on short- and longwave irradiance at the top of the atmosphere and the necessity of accurately representing radiative and other effects of clouds in models, we initiated a project to characterize clouds by high-resolution digital photography looking vertically upward from the surface; the present analysis is limited to thin, boundary-layer cumulus clouds as determined by lidar vertical profiles. Cloud properties are characterized by cloud contribution to zenith radiance. The approach presented here, has the strength of yielding a quantitative measure of the effect of clouds on radiance at the surface and affords the prospect of inverting the measured radiance to infer cloud properties. High spatial resolution is achieved with a commercially available digital camera (Fujifilm FinePix, model S1) having a nominal resolution of $6 \mu \mathrm{rad}$, which, for a cloud 1 $\mathrm{km}$ above the surface, corresponds to a spatial resolution of $6 \mathrm{~mm}$. In addition to resolution, photography from the surface looking upward affords the advantage over satellite imagery in quantitative interpretation of measured signal in that the background radiance is the black of outer space, with contributions to measured radiance only from light scattering by air (Rayleigh scattering), aerosol particles, and cloud drops, with minimal confounding contribution to the signal from surface-leaving radiance.

A Doppler Lidar was used to determined valuable context of the images. The lidar (wavelength 1.5 $\mu \mathrm{m}$; range gate, $30 \mathrm{~m}$; integrating time, $1 \mathrm{~s}$; full angle field of view, $66 \mu \mathrm{rad}$; Pearson et al., 2009) provided time-height profiles of back-scattered intensity and vertical velocity.

A radiation transfer model was used to invert normalized zenith radiance (NZR, zenith radiance per downwelling irradiance) obtained in each of two color channels, red and blue, of the digital images to yield shortwave cloud optical depth (COD, the vertical integral of the scattering coefficient) for clouds of COD less than about 2 and report initial findings to show the capabilities of this approach for characterization of cloud radiance and its variation on fine spatial scales.

Other key measurements were with a multi-filter rotating shadow band radiometer (MFRSR) [Harrison et al., 1994] for cloud detection and aerosol optical depth; and Cimel sun photometer [Holben et al., 1998] for aerosol optical depth.

\section{METHODOLOGY}

The short-range spatial and temporal variation of cloud contribution to zenith radiance has been extensively studied at Brookhaven National Laboratory (Upton, Long Island NY, JuneAugust, 2014), City College of the City University of New York, CCNY/CUNY (New York City, May, 2015; Figure $1 a$ ), and at the Atmospheric Radiation Measurement (ARM) Southern Great Plains (SGP) site in north central 
Oklahoma $\left(36.6^{\circ} \mathrm{N}, 97.5^{\circ} \mathrm{W}\right.$; altitude $317 \mathrm{~m}$, July 2015) (Figure $1 b-e$ ) (Stokes and Schwartz, 1994).

All of these studies revealed frequent occurrence of clouds exhibiting similar short-range structure and frequently consisting of an intimate mixture of blue sky with cloud and/or transmittance of blue through thin clouds. Based on these observations we have reason to believe that the high temporal and spatial variation reported here may be quite general. In this regard we present here one case, selected from the measurements performed at the ARM SGP site on July $31^{\text {st }}, 2015$ (Fig.1, Schwartz et al., 2017).

Our research focus was on optically thin clouds, which are radiatively quite important, (typical local and instantaneous TOA shortwave radiative effect for $\mathrm{COD}=1$, about $80 \mathrm{~W} \mathrm{~m}^{-2}$ ) and are underrepresented in models and analysis. Our quantitative analysis of the images has been restricted thus far to optically thin clouds, optical depth $\lesssim 2$. This low COD value has also been selected to avoid the ambiguity problem noted later in this section (Figure 2).

The cloud layers selected for analysis are indicated by the lidar (Pearson, 2009) in regions of the images (see Figure 1e) of intense return (attenuated backscatter coefficient $\sim 10^{-3.5} \mathrm{sr}^{-1}$ $\mathrm{m}^{-1}$ ), for which the vertical profiles of returned intensity showed a strong peak at cloud altitude 2 $\mathrm{km}$ above the surface. A quantity determined for these clouds was the effective COD, ECOD, which was determined for each image collected with the digital camera, on a pixel-by-pixel basis by interpolation in the relation between COD and NZR shown in Figure 2. The dependence of COD on NZR given in that figure rests on plane-parallel calculations, and thus because of $3 \mathrm{D}$ effects the ECOD so determined for a given pixel may depart from the actual COD, the local vertical integral of the scattering coefficient.

Additionally, the optical depth was also measured by a multifilter rotating shadowband radiometer (MFRSR) and by a Cimel sun photometer. The MFRSR returned signal during much of the period 16:00 - 17:00, with optical depth at $500 \mathrm{~nm} 0.4$ to 0.5 , but with excursions up to 1.5 earlier in the hour and 1.0 at 16:34:00; the high excursions were characterized by low Ångström exponent (0 - 0.2) indicative of optically thin clouds.

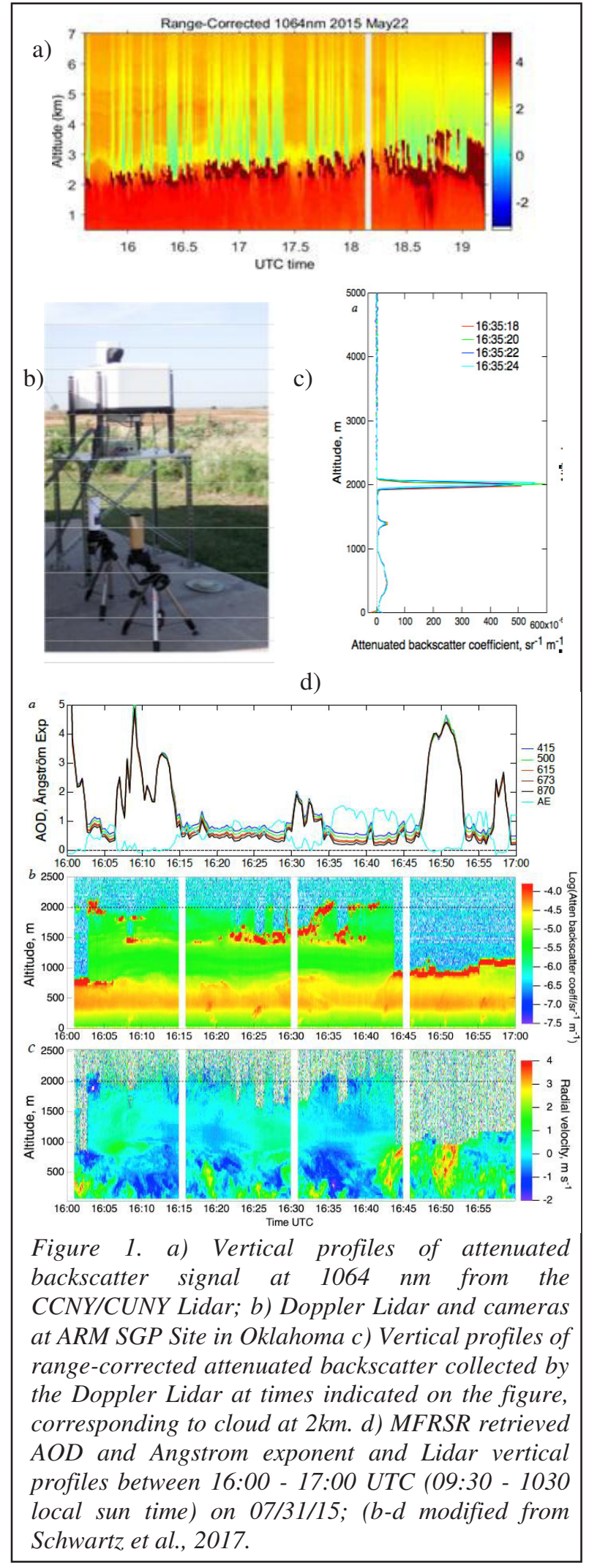

Additional perspective to the atmospheric state was provided by radiosonde (not shown here), 
which showed a region of high relative humidity between 1000 and $2000 \mathrm{~m}$ altitude above the surface, consistent with cloud height determined by lidar. Based on the sounding, the atmosphere is neutral to slightly stable at the altitude of the aerosol and cloud layers, supporting interpretation of the clouds as weakly dissipative, an interpretation supported also by the slightly negative vertical velocities $\left(\sim 1 \mathrm{~m} \mathrm{~s}^{-1}\right)$ indicated by the Doppler Lidar (Schwartz et al., 2017).

The dependence of zenith radiance on COD, shown in Figure 2, was inverted at a given solar zenith angle to yield COD as a function of NZR for 640 and $460 \mathrm{~nm}$, and $\cos (\mathrm{SZA})=0.85$. At low NZR the relation is approximately linear suggesting the possibility of sensitive retrieval of COD from NZR.

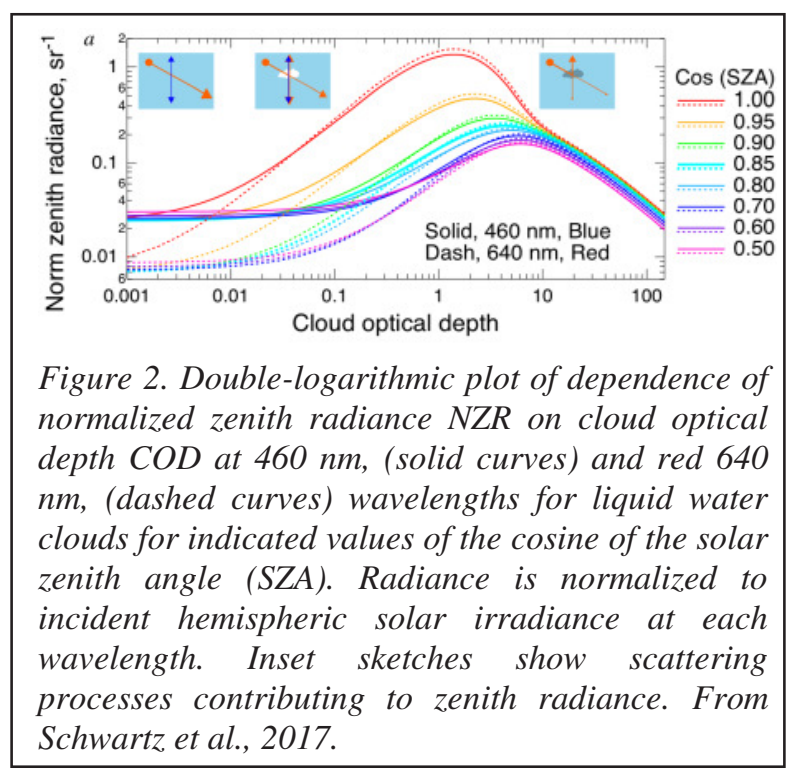

The normalized zenith radiance NZR, denoted $R_{\lambda}^{\mathrm{nZ}}$ (unit: $\mathrm{sr}^{-1}$ ), at a given wavelength $l$ as the ratio of downwelling zenith radiance $I_{\lambda}^{Z}\left(\mu_{0}\right)$ (W $\left.\mathrm{m}^{-2} \mathrm{~nm}^{-1} \mathrm{sr}^{-1}\right)$ to incident downwelling irradiance at the top of the atmosphere ( $\mathrm{W} \mathrm{m}{ }^{-2} \mathrm{~nm}^{-1}$ ), is defined as

$$
R_{\lambda}^{\mathrm{nz}}\left(\mu_{0}\right)=\frac{I_{\lambda}^{\mathrm{Z}}\left(\mu_{0}\right)}{\mu_{0} F_{\lambda}},
$$

where $\mu_{0}$ is the cosine of the solar zenith angle SZA and $F_{\lambda}$ is the direct normal solar irradiance at the top of the atmosphere; this definition differs by a factor of $\pi$ from that of Marshak et al. (2009). Here we specialize to the red and blue channels, having peak sensitivities at 640 and 460 $\mathrm{nm}$, respectively, in the camera employed.
As the inference of COD from NZR is based on a radiative transfer (RT) model that assumes horizontal homogeneity, the retrieval is restricted to clouds that meet this requirement. In the present study use of this model for retrieval of COD gains further justification by the restriction of the retrieval to situations of single, optically thin layer clouds, in which the downwelling radiance is dominated by single scattering, as manifested by the near linearity of the relation between COD and NZR shown in Figure 2 for COD $\lesssim 2$. This restriction also avoids ambiguity arising from the double valued dependence of COD in the retrieval of COD from NZR (so-called ambiguity problem). The lidar profiles confirm this situation in the cases selected.

The normalized zenith radiance $R_{\mathrm{S}}$ is obtained for both the red and blue channels on a pixel-by-pixel basis from the count rate in each pixel as

$$
R_{\mathrm{S}}=R_{\min }+\frac{C-C_{\text {min }}}{C_{\text {max }}-C_{\text {min }}}\left(R_{\text {max }}-R_{\min }\right)
$$

Here $C$ denotes red or blue counts in a given pixel. $C_{\min }$ and $C_{\max }$ denote the minimum and maximum counts, corresponding to cloud-free and maximum-brightness cloud pixels, respectively; determination of $C_{\min }$ and $C_{\max }$ thus requires identification of a nominally cloud-free region and of a region of maximum cloud brightness, respectively, in a single image or in temporally proximate images. $R_{\min }$ and $R_{\max }$ similarly denote the NZR determined from the RT calculations in the absence of cloud and at the maximum brightness as COD is increased, respectively, at the pertinent solar zenith angle. The calibration obtained in this way is expected to be transferrable to temporally proximate images with approximately constant solar geometry. Using a cloud-free region in the image itself to determine $C_{\min }$ implicitly accounts for contribution to zenith radiance from aerosols, the contributions to radiance from clouds and aerosols being nearly additive at the low optical depths for which the method is applicable. The ability to determine NZR from the camera counts for each pixel (and separately for the red and blue channels) in turn permits determination of COD from the data for each channel on a pixel-by-pixel basis. 


\section{RESULTS}

The lidar measurements for the day of analysis indicate that the Planetary Boundary Layer signal was temporally much more uniform than that from

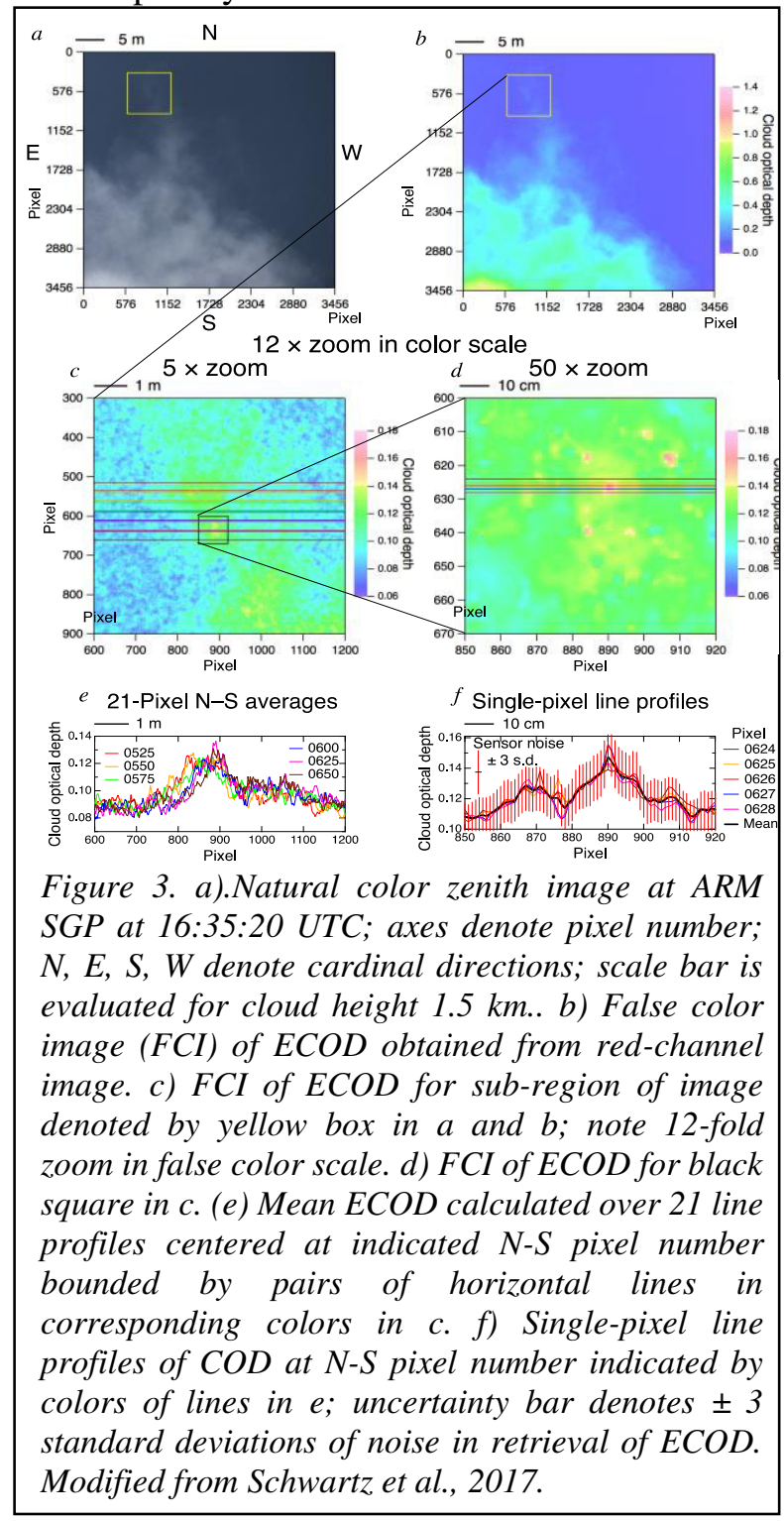

the clouds, consistent with an aerosol layer (or nascent cloud layer) at about $1.5 \mathrm{~km}$ that briefly developed into a thin cloud; the slight variation in aerosol signal, which dominated the return over the 16:35 - 16:40 period (Figure $1 c \& d$ ) is attributed more to variation in relative humidity than to variation in aerosol amount. We analyzed situations where the transmittance through the cloud layers was sufficiently high that it can be inferred that the return from above $2 \mathrm{~km}$ is indicative of the absence of any significant aerosol or clouds above $2 \mathrm{~km}$; this conclusion is also supported by the sounding. An image that satisfied all these requirements, taken at 16:35:20 and examined in Figure 3, exhibits considerable variation in ECOD on a variety of spatial scales. We find that such variation in radiance at all scales examined, with coherent spatial structures at scales as low as $10 \mathrm{~cm}$. We attribute this variation to variation in cloud optical depth (Figure $3 f$ ) on such scales, that we hypothesize to be due to local turbulence. Should this turn out to be the case, then it suggests that high resolution imaging of clouds from the surface may be a means of remotely examining the variation of such turbulence at scales approaching the Kolmogorov scale. Such imaging may thus provide an approach to relating these variations to atmospheric turbulence, which we hypothesize is responsible for the fluctuations in radiance observed on the centimeter scale.

\section{CONCLUSIONS AND FUTURE WORK}

A method is presented here to infer cloud optical depth COD on a pixel-by-pixel from highresolution digital photographic images by scaling the counts in individual pixels to COD via RT calculations. We find that there is commonly considerable variation in radiance at all scales examined, with coherent spatial structures at scales as low as $10 \mathrm{~cm}$. Future work will include further validation and comparison based on lidar retrieved COD.

\section{References}

[1] Pearson, G., F. Davies, and C. Collier, 2009: An Analysis of the Performance of the UFAM Pulsed Doppler Lidar for Observing the Boundary Layer, J. Atmos. Ocean. Tech., 26(2), 240-250

[2] Stokes, G. M., and S. E. Schwartz, 1994, The Atmospheric Radiation Measurement (ARM) Program: Programmatic Background and Design of the Cloud and Radiation Test Bed, Bull. Amer. Meteorol. Soc., 75(7), 1201-1221

[3] Schwartz S. E., Huang D. and Vladutescu D. V. 2017: High-Resolution Photography of Clouds from the Surface: Retrieval of Optical Depth of Thin Clouds down to Centimeter Scales. J. Geophys. Res. - Atmos., Paper \#2016JD025384, in press

[4] Marshak, A., Y. Knyazikhin, J. C. Chiu, and W. J. Wiscombe (2009), Spectral invariant behavior of zenith radiance around cloud edges observed by ARM SWS, Geophys. Res. Lett., 36, L16802 